\title{
Study of Energy Management in Wireless Visual Sensor Networks
}

\author{
Shuruq Abboud Hashim ${ }^{1}$, Dr. Muna Mohammed Jawad ${ }^{2}$, Dr. Bassam M.S Wheedd ${ }^{3}$ \\ Control \& System engineering department, University of Technology, Baghdad, Iraq. \\ 61272@student.uotechnology.edu.iq,120012@uotechnology.edu.iq,60164@uotechnology.edu.iq
}

\begin{abstract}
Wireless Visual Sensor Networks (WVSNs) are a branch of Wireless Sensor Networks (WSNs), WVSN nodes vary from standard WSN nodes in the ability of sensing the environment in two dimensions rather than in one. Therefore, it follows the three main fundamentals of WSNs: wireless networking, distributed sensing and low power hardware. This paper discusses different challenges that face the design of WVSNs like deployment of nodes, field of view overlapping, image analysis, area coverage and energy consumption. Efforts have been done mainly to survey the problem of energy consumption that can affect the lifetime of visual sensor network and overview the different techniques that have been used by many researchers to handle this crucial issue.
\end{abstract}

Index Terms-Wireless visual sensor networks; Field of view (FOV); Image analysis; Energy consumption; Deployment; Coverage.

\section{INTRODUCTION}

Wireless sensor networks (WSNs) are considered as an overturned technology that can modify the process of collecting data from the environment and interact with a real world as the same form that mobile communication altered the manner of people communication.

WSNs connect a massive number of nodes, each of which has the ability of sensing, computing, and wireless communicating. The task of WSNs is to cover the environment and gather particular data about it [1].

WSNs had pulled towards a considerable attention of the researchers in the last decade; most of them had focused on WSNs that gather scalar data like, humidity, vibration, pressure and temperature. Scalar data may be inadequate for some implementations like traffic monitoring and automated surveillance. Therefore, the necessity grows for WSNs with sensors of many dimensions measurements like camera sensors that we mention as Wireless Visual Sensor Networks (WVSNs). Smart camera sensors gather visional data, which are prosperous of information and present enormous strength when utilized in WSNS [2].

A $W V S N$ is composed of many nodes, every node is provided with a low energy embedded processor, power source, image sensor, and a wireless transceiver for communication. These nodes must interact with the network base station or sink where data is collected and processed for the consumption of end users [3]. In WVSNs, as shown in Fig. (1), an enormous number of camera nodes compose a distributed system. The camera sensor nodes have the ability to process image data locally, excerpt pertinent data, handle certain tasks with other cameras on the implementation, and submit the end user with wealthy descriptions of captured incidents. WVSNs are in many, one of a kind and more difficult compared to other WSNs.

According to swift development of WVSNs, many applications have been carried out such as Surveillance of public places, remote areas and parking lots, Environmental monitoring of dangerous areas, building monitoring, animal habitats, Smart homes of elderly care and Teleconferencing smart meeting rooms [4]. This paper demonstrates the main traits of WVSNS and the challenging issues of a good WVSN design such as camera coverage, energy consumption, deployment, etc. and then demonstrates proposed 
solutions for energy consumption problem in WVSNs, so that to expand the network lifetime. The rest of the paper is arranged as follows: Section II argues the relevant works in WVSNs; section III discusses some challenging issues in WVSNs; section IV categorizes proposed solutions to energy consumption problem; and section $\mathrm{V}$ presents conclusions.

\section{RELATED WORKS}

Long before, many researchers have been addressing essential issues of visual sensor network. Ian F. Akyildiz, et al., [5] discussed the state of the art and the major research challenges in architectures, protocols and algorithms for wireless multimedia sensor networks. Jennifer Yick, et al., [1] presented a top-down approach to survey different protocols and algorithms proposed in recent years. They compared and contrasted the different kinds of wireless sensor networks and summarized the current sensor techniques. Charfi, et al., [2] highlighted the challenges and possibilities of WVSNs, and tackled the main issues of WVSNs studies, in particular camera coverage enhancement, network architecture, low power visual data processing and communication and identified conceivable method in this field. Stanislava Soro and Wendi Heinzelman, [4] gave a general review of the trend state of the art in the domain of WVSNs, by discussing some pertinent research aspects. M. Karakaya and H. Qi, [6] tackled the problem of communication and energy efficiency in WVSNs. They presented that all sensor nodes transmit very controlled amount of data and a limited number of camera nodes are involved in. Rault, et al., [7] presented a top-down survey of the trade-off between application requirements and lifetime extension that arise when designing WSNs. A. C. Bhatnagar, et al., [8] suggested a power efficient gathering in sensor information system, a control method to select the adequate subset of expanded camera sensors for providing the desired density of nodes in the detecting mode. Rajkumar, et al., [9] surveyed the different research challenges in WSNs. We give an overview of major research issues of WVSNs, specifically the challenges face design concentrating on energy consumption problem with proposed solution to it.

\section{CHALLENGING ISSUES in WVSNS}

\section{A. AREA COVERAGE}

Coverage problem in WVSNs is more complicated as compared to coverage problem in WSNs. It is coverage based, target based, connectivity and lifetime. The camera's orientation has an effective role in the coverage problem of WVSNs. The orientation determines the field of view (FOV) and it is a major factor for the coverage of the network. Fig. (2) Displays view field of a camera in the WVSN. When the camera's orientation is changed, the coverage area of the WVSN is also changed. If cameras are properly mounted, a large area can be covered using a minimum number of them. The visual monitoring of the target area relies on the nodes' posture, resolution and orientation of the camera [10], for a firm coverage of an area, the cameras must be mounted with fixed focal lens. The nodes in WSNs can collect data only in its sensing range while cameras in WVSNs can remotely capture image.

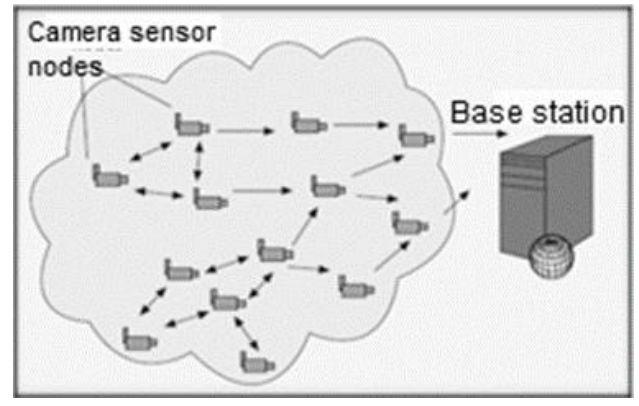

Fig. (1) Wireless VisuAl SENSOR NETWORK [4].

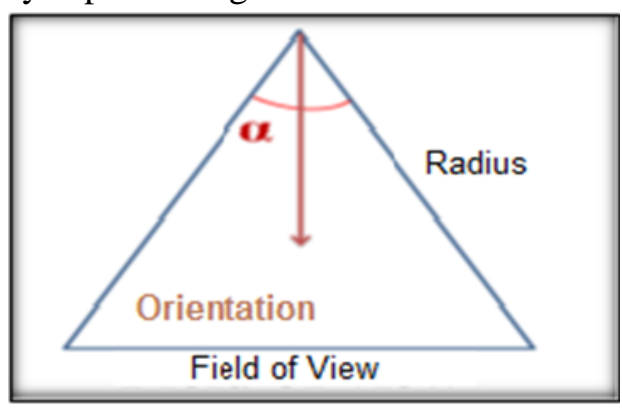

Fig. (2) Field OF VIEW OF CAMERA [10]. 


\section{B. DEPLOYMENT STRATEGY}

There are Two ways of cameras deployment in WVSNs: random and controlled. To cover a wide area, immoderate number of directional cameras needed. In a random deployment, camera sensors will be scattered, and visual sensor nodes densely or lightly cover some sections of area. Nodes could be dropped from aircraft or released over the ocean with respect to the characteristics of the area to be covered and the requirements of the application. The process of random deployment may lead to decay of camera-enabled sensors, producing a decay case. A decay could damage the monitoring capability of WVSNs. The failures in source nodes could decrease the quantity of visual data that move to the sink node, possibly decreasing the observation quality of monitoring applications. The sensors locations and camera orientations in random deployment cannot be predicted. Therefore, an enormous number of nodes is deployed to prevent any limitation. In a controlled deployment, sensors may be properly positioned in a pre-processed design permits the covered region to be maximized using the least number of sensors, theoretically enabling effective arrangement of excrescent nodes for a high degree of availability [11].

\section{FIELD OF VIEW OVERLAPPING}

In case of immoderate deployment of sensor nodes, $F o V$ of the sensors may be overlapped. If the $F o V$ of two or more number of sensor cameras intersects, more than one visual sensor can capture the identical target from various directions. Some sensors with overlapped sensing areas keep an eye on the same object target, but from different sights, therefore they earn various data Fig. (3), shows the overlapped FoV [10].

\section{OCCLUSION}

While implementing visual monitoring, the $F o V$ of the sensors could be obstructed unexpectedly by several moving obstacles. If an obstacle is persistent, the visual sensor will be unsuitable for the surveillance purposes of the application. Generally, occlusion is unforeseeable and could minimize the monitoring field of directional camera sensors. Fig. (4), shows a target is covered by an obstacle and generates occlusion in the view of cameras while carrying out visual monitoring [10].

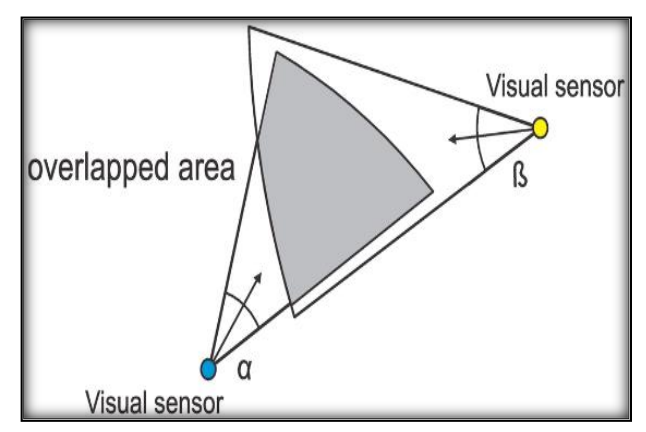

FIG. (3) CAMERA COVERAGE IN WVSNS SENSOR [10].

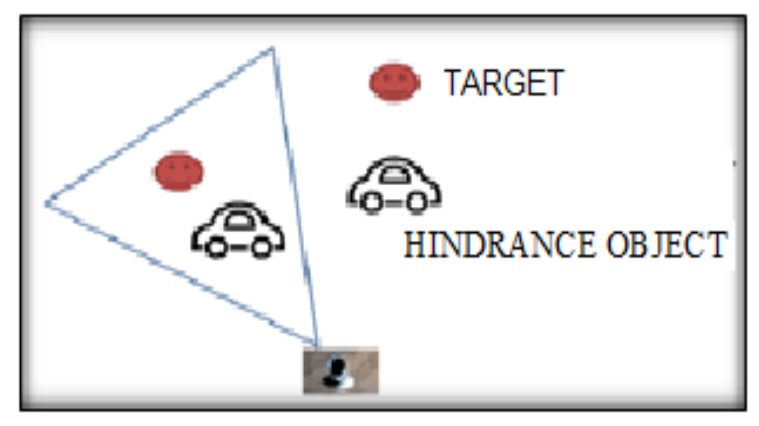

FIG. (4) OCCLUSION IN THE VIEW OF CAMERA WITH OVERLAPPED FOV [12].

\section{E. ENERGY CONSUMPTION}

One of the important considerations in WVSNs design is energy consumption, it is an essential interest in WVSNs more than in standard WSNs. Actually; sensors are battery-limited devices, while multimedia applications generate large amount of data, which need high rates of transmission and spend most of their energy for two major jobs: sensing and processing [13]. The energy consumption of traditional sensor nodes is governed by communication; this may not be true in WVSNs. Consequently, algorithms, architectures and protocols are used to expand the network lifetime while outfitting the quality of services $Q o S$ needed by the application are a critical problem. [5]. 


\section{PROPOSED SOLUTIONS FOR ENERGY CONSUMPTION in WVSNS}

A need arises to improve the hardware and software solutions in order to be power efficient. Energy consumption is proportionate to the total amount of work a WVSN node achieves [13].

As known, spatial data needs more number of bits than scalar data like motion, temperature and pressure. It makes transmission of graphic data stricter. Such a way, the number of working sensors will be concerned with the network lifetime directly and more energy is consumed due to big data [10]. Energy saving techniques can be classified as follows:

\section{Sensor wake up scheduling mechanism to switch between active and sleep states [14].}

This approach can be accomplished using node redundancy [15]. Many essential issues must be ken into consideration and these are:

1. The first issue focuses on the principle every node must follow to define whether to step inside sleep state. The probable factors that affect are:
a. Energy previously consumed.
b. Remaining energy resources.
c. The role in the sensing functions.
d. The sensors nature.

2. The second issue is the time needed by those nodes to make a decision. Nodes can use counters; they can follow up a schedule or observe the running power resources and coverage of the targets

3. The third issue is the period a sensor has to stay in the sleep state. The modeled solution can place nodes to sleep for a particular time, in addition to, it can assume that nodes check the adjacent seeking energy depleted or failed nodes, or wait for external incidents.

4. At last, the fourth issue concerning the nature of the sleeping mode. As matter of fact, a sleeping sensor may be in a low-energy operation allowing communication but with none sensing functioning. Other approach is to permit cyclic sensing of low frequency. Other option is to slake the sensing and communication units, implementing locally an algorithm to compute the time until node reactivation. [16].

For real world environments; the coverage maximization, Connectivity preservation and power saving can be carried out in various methods and they can be preferred over the others. It influences the selection of nodes to sensing or routing tasks.

Finding redundant nodes is one of the most essential aspects of using redundancy to preserve energy. In normal WSNs, the omnidirectional sensing nature points out those adjacent nodes are probable to gather the same basic data; this is not always true for directional sensors, because near visual sensors may not recapture the identical visual data according to theirs directions or obstruction. Two nodes are depicted as adjacent if they have overlapping $F o V$, and it is tested via some algorithms from computer vision domain [17].

Many protocols and algorithms to expand the life of the network by conserving energy in WVSNs, while maintaining connectivity and coverage if there are redundant nodes, some of these are:

1. Ai and Abouzeid [18] suggested the Sensing Neighborhood Cooperative Sleeping \{SNCS\} protocol uses the remaining energy of every node as a primacy for stepping nodes into sleep state. The sleeping nodes turn into active state when their resources energy exceed the remaining energy of existent active nodes.

2. Zamora et al. [19] illustrated the nodes that cannot view the surveilled target turn to sleep state and is being self-activated after a limited sleep time. Nodes are able to interchange messages pointing out the immediate and previous views of the cameras. Data like that is utilized to send off nodes into sleep state, possibly expanding lifetime of the network.

3. Cai et al. [20] proposed a sub combination of sensors for target covering with single sensors sharing in one or more cover sets. One and only subset of sensors are activated at each time, conserving energy through deactivating the residual sets. 
4. Pescaru et al. [21] presented algorithms that switch off less important excessive nodes. Every active node estimates its power and if it is less than a threshold, a redundant adjacent node, which is in sleep state, is switched on.

5. Istin et al. [22] clarified that nodes that reveal FOV loss apprise the nearer nodes. According to the response, the node recognizes the ideal cameras, which must be turned on. When the barriers pass by and the main FOV is restored, the prime node informs the adjacent cameras that presented, the former demand that they have to switch off.

2. Energy management through adjusting the transmission range of sensor nodes [14].

Zamora \& Marculescu [23] found distributed power management of camera nodes depending on symmetrical node wake-ups to decrease the power usage of cameras sensors. They proposed a policy assumes that every sensor node is awake for a particular interval, and then the camera node chooses if it has to step inside the low energy state relied on the timeout statuses of its neighboring nodes. Camera nodes can alternatively do so based on polling from other neighboring cameras.

Data transmission techniques in WVSNs can be divided into three categories as follows:

a. Image _video transmission through single hop techniques.

It is found that in the first category, the job of Lecuire et al. [24], Who introduced a wavelet image transmission mechanism in WVSNs relied on decomposing an original image applying " a discrete wavelet transform $D W T$ " and separated it into packets of various priorities. The transmission begins with packets of high priority, and a node only sends subsequent packets if its battery standard is over a particular threshold. Such a mechanism sacrifices a specific quantity of rebuilding quality to extend the WVSN's life. Yu et al. [25] presented a scheme for "JPEG 2000" image transmission over WVSNS that reduces power consumed while satisfying quality of service "QoS" guarantees. Both [24], [25] used "wavelet image compression" to outfit resolution scalability.

b. Multi hop transmission where the transmission strategy is determined on a "hop-by-hop" basis.

The work of $\mathrm{Wu}$ and Abouzeid [26] is a representative example in the second category, who presented a "hop-by-hop" reliability strategy, which does not optimize the "end-to-end" performance and put in extra power cost and delay because of extra processing.

c. "End- to- end" multipath transmission techniques [2].

Many works in the third category merge path diversification and error correcting codes to provide "end-to-end" reliability in multi hop networks, where multiple transmission paths used to rise reliability. Algorithms of finding the number of channel packets and the transmission paths that enhance the reliability/energy cost trade off had been proposed in [27], [28].

\section{Energy-efficient data aggregation, gathering and routing. [14]}

Data aggregation technique is the operation of gathering data arriving from various adjacent sensor nodes and bringing out redundancy from collected data then send it to base station with adequate routing technique, thereby enhancing the total network performance and prolonging the lifetime [29].

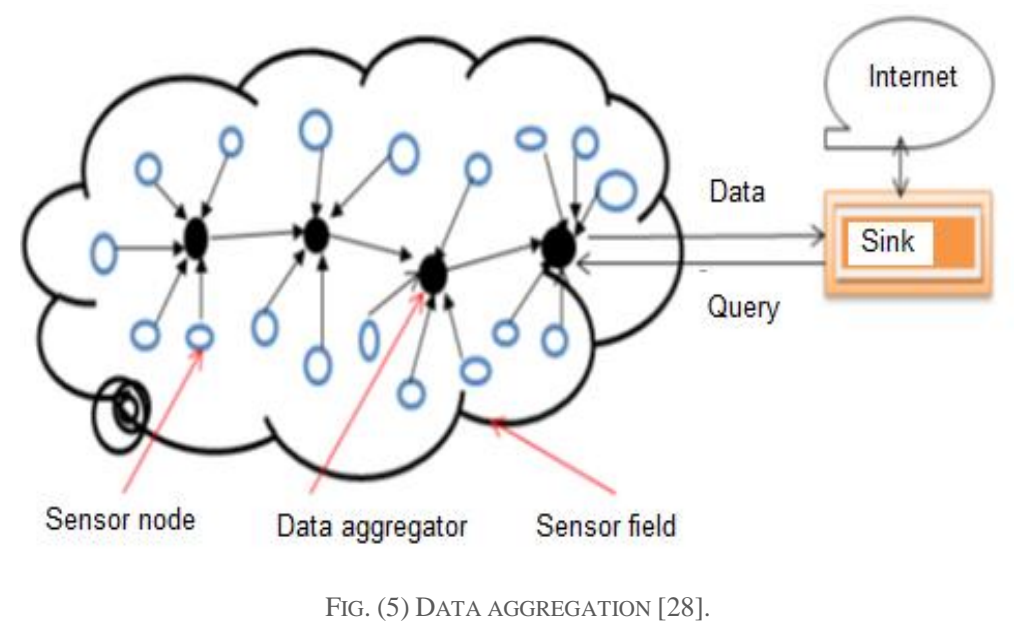

Received 29 July 2019; Accepted 8 October 2019 
Hüseyin Akcan et al; [30] presented a new deterministic data aggregation method for WSNs. This method utilizes a deterministic sampling method comparing to random sampling. The size of the outcome sample is so slight and needs less transmission energy, which results in expanding network life. Yang Xiao et al; [31] also used data compression as a collecting method in WSNs using piecewise approximation algorithm compressing data. Due to the reduced size of packet, hence the network lifetime is extended. The disadvantage of this algorithm is the loss of data. Ultimate energy efficient routing protocols in WVSNs utilizes shortened route or multi-path routing techniques to tackle end-to-end delay and/or bandwidth. Examples of protocols intended specifically for routing WVSNs video streams. A modified version of Low Energy Adaptive Clustered Hierarchy $\{L E A C H\}$ is described as a hierarchical protocol to minimize video deformation and tackle bandwidth restrictions, Ehsan and Hamdaoui [32]. The sensor nodes are divided into clusters in LEACH protocols, and then the sensor node with higher resources is chosen as the cluster head $(\mathrm{CH})$ [33].

Kandris et al's routing scheme; [34] combines an energy-efficient routing protocol with a video packetscheduling algorithm. The suggested hierarchical routing protocol will select paths and manage network load depending on sensor node residual power.

It is conceivable to accomplish less consumption of energy or communication costs if the data is being sensed are interconnected by means of applying the information theory using a combination of network coding $\{N C\}$ and distributed source coding $\{D S C\}$. Li et al. [35] suggested a coding and routing technique .They applied an analytic "power_rate_distortion" model $\{P-R-D\}$ for distributed video coding $\{D V C\}$ to minimize deformation of video and expand the life of the network.

\section{CONCLUSION}

WVSNs are the following generation of WSNs allowing visual application based on data. WVSNs are advantageous in different types of applications. In this paper, we have shed light on the major challenges of wireless visual sensor networks and focus specifically on the energy consumption problem as a main factor that influences WVSNs design. It is found that many researches on energy consumption are just at the beginning and power cost is a main study issue in WVSNs.

The visual data is greater and more entangled than standard data. It is known that more resources are consumed through operations of processing and transferring of visual data in addition to process of data rebuilding at the sink node counts on the collaboration among numerous connected data sources nodes to remodel the entire form of the concerned objects. These recent difficulties make the algorithms and protocols of the traditional WSN unsuitable to WVSNs. Current studies in WVSNS are still at the beginning and till now there are many open topics need to be evaluated in the direction of energy consumption, visual data sensing, processing and transmission.

\section{REFERENCES}

[1] J. Yick, B. Mukherjee, and D. Ghosal, "Wireless sensor network survey," Comput. Networks, vol. 52, no. 12, pp. 22922330, 2008.

[2] Y. Charfi, N. Wakamiya, and M. Murata, "Challenging issues in visual sensor networks," IEEE Wirel. Commun., vol. 16, no. 2, pp. 44-49, 2009.

[3] A. Marcus and O. Marques, "An eye on visual sensor networks," IEEE Potentials, vol. 31, no. 2, pp. 38-43, 2012.

[4] S. Soro and W. Heinzelman, "A Survey of Visual Sensor Networks," Adv. Multimed., vol. 2009, pp. 1-21, 2009.

[5] F. Akyildiz, T. Melodia and K. R. Chowdury, "Wireless multimedia sensor networks: A survey," in IEEE Wireless Communications, vol. 14, no. 6, pp. 32-39, December 2007.

[6] M. Karakaya and H. Qi, "COMMUNICATION AND ENERGY EFFICIENCY IN WIRELESS VISUAL SENSOR NETWORKS FOR PEOPLE LOCALIZATION,” in conference: Future of Instrumentation International Workshop (FIIW), 2012.

[7] Tifenn Rault, Abdelmadjid Bouabdallah, Yacine Challal. "Energy Efficiency in Wireless Sensor Networks: a top-down survey". Computer Networks, Elsevier, 2014, 67 (4), pp.104-122. 
[8] A. C. Bhavnagar, R. Varshney and K. Manu, "ENERGY EFFICIENCY IN WIRELESS VISUAL SENSOR NETWORKS USING PEGASIS," International Research Journal of Engineering and Technology (IRJET), Volume: 04 Issue: 05 | May -2017

[9] Rajkumar, Dr. H G Chandrakanth, Dr. D G Anand, and Dr. T John peter "RESEARCH CHALLENGES AND CHARACTERISTIC FEATURES IN WIRELESS SENSOR NETWORKS," Int. J. Advanced Networking and Applications, 2017.

[10] Rajkumar, Dr. H G Chandrakanth, Dr. D G Anand, and Dr. T John peter "RESEARCH CHALLENGES AND CHARACTERISTIC FEATURES IN WIRELESS SENSOR NETWORKS," Int. J. Advanced Networking and Applications, 2017.

[11] L. Liu, H. Ma and X. Zhang, "ON DIRECTIONAL K-COVERAGE ANALYSIS OF RANDOMLY DEPLOYED CAMERA SENSOR NETWORKS," IEEE International Conference on Communications, 2008.

[12] D. G. Costa, I. Silva, L. A. Guedes, and F. Vasques, "Availability Issues in Wireless Visual Sensor Networks,” pp. 2795-2821, 2014.

[13] S. Farzana, K. A. Papry, A. Rahman, and R. Rab, "Maximally pair-wise disjoint set covers for directional sensors in visual sensor networks," IFIP Wirel. Days, vol. 2016-April, no. iv, 2016.

[14] M. F. Abdulhalim and A. Attea, "Multi-layer Genetic Algorithm for Maximum Disjoint Reliable Set Covers Problem in Wireless Sensor Networks," 2004.

[15] Daniel G. Costa, Francisco Vasques, Paulo Portugal " ENHANCING THE AVAILABILITY OF WIRELESS VISUAL SENSOR NETWORKS: SELECTING REDUNDANT NODES IN NETWORKS WITH OCCLUSION", Applied Mathematical Modelling, 2016.

[16] M. Cardei and J. Wu, "Energy-efficient coverage problems in wireless ad-hoc sensor networks," vol. 29, pp. 413-420, 2006.

[17] D. G. Costa and L. A. Guedes, "The coverage problem in video-based wireless sensor networks: A survey," Sensors, vol. 10, no. 9, pp. 8215-8247, 2010.

[18] J. Ai and A. A. Abouzeid, "Coverage by directional sensors in randomly deployed wireless sensor networks," pp. 2005-2006, 2006.

[19] Zamora, N.H.; Kao, J.C.; Marculescu, R. "DISTRIBUTED POWER-MANAGEMENT TECHNIQUES FOR WIRELESS NETWORK VIDEO SYSTEMS". In Proceedings of the Conference on Design, Automation and Test in Europe, Nice, France, April 2007.

[20] Cai, Y.; Lou, W.; Li, M.; Li, X.Y. "TARGET-ORIENTED SCHEDULING IN DIRECTIONAL SENSOR NETWORKS". In Proceedings of IEEE Infocom, Anchorage, AK, USA, May 6-12, 2007.

[21] D. Pescaru, C. Istin, D. Curiac, and A. Doboli, "Energy saving strategy for video-based wireless sensor networks under field coverage preservation," 2008 IEEE Int. Conf. Autom. Qual. Testing, Robot. AQTR 2008 - THETA 16th Ed. - Proc., vol. 1, pp. 289-294, 2008.

[22] C. Istin, D. Pescaru, H. Ciocarlie, D. Curiae, and A. Doboli, "Reliable field of view coverage in video-camera based wireless networks for traffic management applications," Proc. 8th IEEE Int. Symp. Signal Process. Inf. Technol. ISSPIT 2008, pp. 63-68, 2008.

[23] N. H. Zamora and R. Marculescu, "Coordinated distributed power management with video sensor networks: Analysis, simulation, and prototyping," 2007 1st ACM/IEEE Int. Conf. Distrib. Smart Cameras, ICDSC, pp. 4-11, 2007.

[24] V. Lecuire, C. Duran-Faundez, and N. Krommenacker, "Energy-efficient transmission of wavelet-based images in wireless sensor networks,” Eurasip J. Image Video Process., vol. 2007, 2007.

[25] W. Yu, Z. Sahinoglu, and A. Vetro, "Energy efficient JPEG 2000 image transmission over wireless sensor networks," GLOBECOM - IEEE Glob. Telecommun. Conf., vol. 5, pp. 2738-2743, 2004.

[26] H. Wu and A. A. Abouzeid, "Error resilient image transport in wireless sensor networks," Comput. Networks, vol. 50, no. 15 , pp. 2873-2887, 2006.

[27] P. Djukic and S. Valaee, "Minimum Energy Reliable Multipath Ad Hoc Networks,” no. June 2004, pp. 5-7, 2017.

[28] Y. Charfi, N. Wakamiya, and M. Murata, "Trade-off between reliability and energy cost for content-rich data transmission in wireless sensor networks," 2006 3rd Int. Conf. Broadband Commun. Networks Syst. BROADNETS 2006, 2006.

[29] Y. Somkuwar and S. D. Kamble, "Review on: Data Aggregation and Dynamic Routing in Wireless Sensor Network," Int. J. Comput. Appl., vol. 88, no. 17, pp. 31-35, 2014.

[30] H. Akcan and H. Brönnimann, "A new deterministic data aggregation method for wireless sensor networks," Signal Processing, vol. 87, no. 12, pp. 2965-2977, 2007.

[31] X. F. Yang, X. B. Wu, and J. A. Huang, "TAGPP: A tiny aggregation algorithm with preprocessing in local cluster," Proc. - Int. Conf. Networks Secur. Wirel. Commun. Trust. Comput. NSWCTC 2009, vol. 2, pp. 390-393, 2009.

[32] S. Ehsan and B. Hamdaoui, "A survey on energy-efficient routing techniques with QoS assurances for wireless multimedia sensor networks," IEEE Commun. Surv. Tutorials, vol. 14, no. 2, pp. 265-278, 2012. 
[33] R. A. Hamamreh, “An Energy-Efficient Clustering Routing Protocol for WSN based on MRHC," Int. J. Digit. Inf. Wirel. Commun., vol. 8, no. 3, pp. 214-222, 2018.

[34] D. Kandris, M. Tsagkaropoulos, I. Politis, A. Tzes, and S. Kotsopoulos, "Energy efficient and perceived QoS aware video routing over Wireless Multimedia Sensor Networks," Ad Hoc Networks, vol. 9, no. 4, pp. 591-607, 2011.

[35] C. Li, J. Zou, H. Xiong, and C. W. Chen, "Joint coding/routing optimization for distributed video sources in wireless visual sensor networks," IEEE Trans. Circuits Syst. Video Technol., vol. 21, no. 2, pp. 141-155, 2011. 